\title{
Multimodal composition of the digital patient: a strategy for the knee articulation
}

\author{
Hon Fai Choi - Andra Chincisan - Matthias Becker • \\ Nadia Magnenat-Thalmann
}

Published online: 8 May 2014

(C) Springer-Verlag Berlin Heidelberg 2014

\begin{abstract}
Creating virtual bodies of real patients and using them for diagnosis and treatment planning offer the potential to further empower clinical decision making by medical experts. Virtual patient modeling allows to examine the mechanical and physiological conditions under which articulations are operating in a variety of activities without putting the patient in hazard. The continuous scientific progress has led to an increased range of musculoskeletal data and knowledge being available, covering multiple scales of the musculoskeletal system. A fuller integration of these modalities can broaden the scientific basis of virtual articulation modeling in patients, but poses challenges for data fusion and coupling of simulations. Here, we present a multimodal strategy to compose virtual models of the knee articulation based on a complementary spectrum of data that enables simulations on different scales.
\end{abstract}

Keywords Virtual human - Medical imaging ·

Segmentation $\cdot$ Multiscale modeling

\section{Introduction}

Clinical examinations and treatment planning for musculoskeletal disorders (MSD) predominantly depend on the

\author{
H. F. Choi $(\varangle) \cdot$ A. Chincisan · M. Becker · N. Magnenat-Thalmann \\ MIRALab, University of Geneva, Geneva, Switzerland \\ e-mail: choi@miralab.ch \\ A. Chincisan \\ e-mail: chincisan@miralab.ch \\ M. Becker \\ e-mail: becker@miralab.ch \\ N. Magnenat-Thalmann \\ e-mail: thalmann@miralab.ch
}

physician's personnel experience, training and interpretation of general guidelines. Medical assessment is often based on subjective scoring indices [1], which often does not allow to reliably differentiate underlying causes. Therefore, further investigation and development of quantitative and patientspecific methodologies to extract deeper information from medical data are of paramount importance. This is particularly compelling for the knee, which is the largest weightbearing joint in the human body, sustaining relative large loads even during daily ambulatory activities. Moreover, the articulation surfaces are incongruent, such that the dynamics and stability are heavily dependent on the mechanical synergy of the soft tissues, making the knee joint particularly susceptible to injury and arthritis [2].

Virtual patient modeling using principles of mechanics can help provide a deductive means not only to better understand MSD but also to assist clinicians. In order to ensure that the assessment is pertinent to the patient's case, construction of the virtual model should rely on personalized medical data as much as possible. However, it is a challenging endeavor to process all data modalities and individualize all aspects of the musculoskeletal system in a single modeling framework. Therefore, modeling approaches are often focused on the input of the modality that is most relevant for the application envisioned, while relying on approximations to substitute for other aspects. Only recently have modeling workflows emerged that incorporate a multimodal strategy to maximize the subject-specific content by exploiting multiple modalities simultaneously $[3,4]$. However, the advancement of musculoskeletal research has resulted in an expansion of data acquisition methods that can further empower virtual patient modeling, ranging from imaging techniques that allow for functional examination [5] to microscale tissue testing and cellular characterization of MSD [6]. Therefore, the motivation for this paper is to present a modeling 
Fig. 1 Overview of the multimodal workflow combining subject-specific (blue) and experimental (green) data to construct models on different levels as input to macro- and microscale simulations
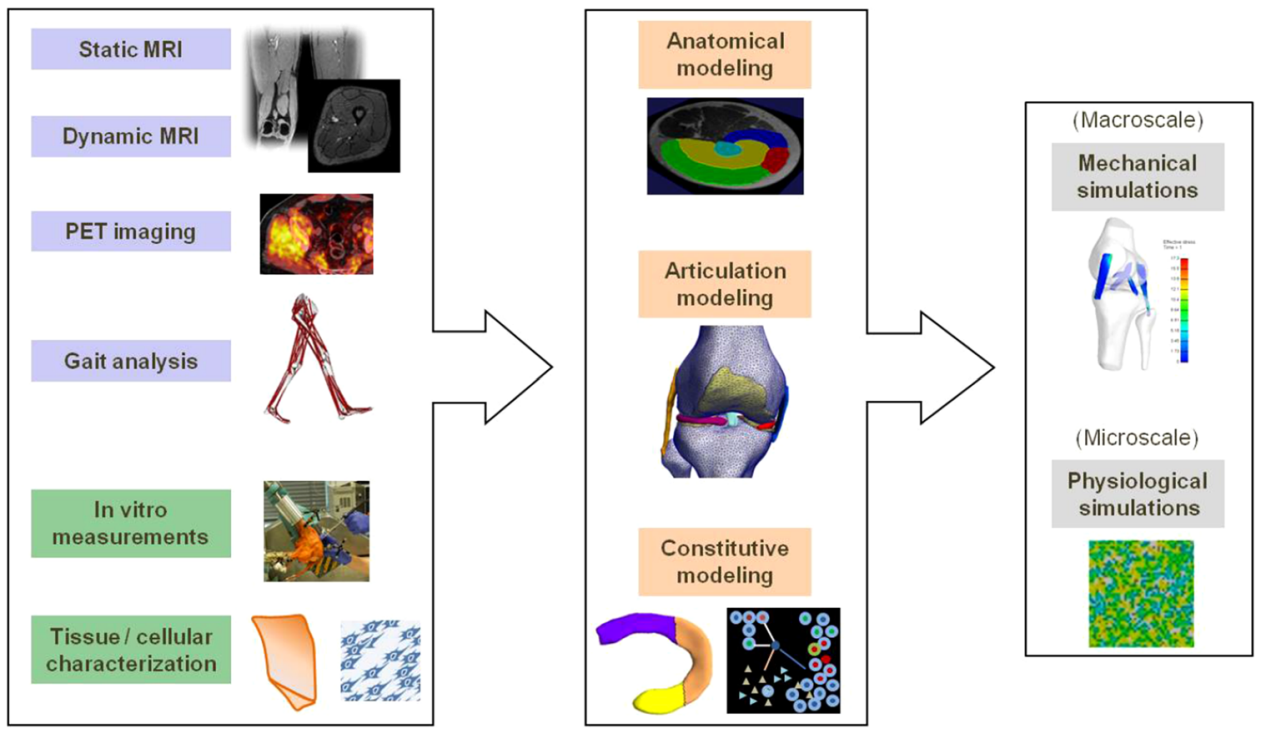

workflow focusing on the knee articulation that takes further advantage of the wide spectrum of available musculoskeletal data, allowing to explore simulations on different scales as illustrated in Fig. 1. In the following, the existing work is first broadly reviewed, followed by a description of the methodological integration in the proposed multimodal workflow. Then, a prospective case study demonstrating the value for medical application is presented. Future developments and perspectives are discussed in the conclusion.

\section{Related work}

\subsection{Anatomical modeling}

Medical imaging has become a cornerstone in clinical routine practice, requiring robust computational segmentation procedures for morphological analysis. Deformable models play an important role in medical image segmentation due to their flexibility, robustness and the possibility to adapt model properties. Bredno et al. [7] provide an overview of the typical structure, common components and a discussion of common pitfalls of deformable models. A survey of medical image segmentation using deformable models can be found in, e.g. [8].

An approach to increase robustness and correctness is to perform segmentation based on multi-channel images. For example, Chang et al. [9] presented an active contour model for vector images and demonstrated it on RGB color images. Similar concepts can be translated to procedures for multichannel magnetic resonance imaging (MRI) segmentation, for which early work has considered the combination of $T_{1}$, $T_{2}$ and proton density (PD) weighted images. More recently, Angelini et al. [10] have devised a statistical hybrid approach which was demonstrated with the Visible Human data set and multi-protocol MRI volumes of the brain. Pauly et al. have used regression and multi-atlas approaches for multi-channel dixon images for organ localization, while segmentation of MS lesions in multi-channel MR images based on spatial decision forests was considered by Geremia et al. [11].

For musculoskeletal applications, muscle segmentation in MRI images has proven to be a particularly complex task because of the low contrast between adjacent muscles. Existing approaches all invoke prior knowledge as a statistical model to account for inter-subject variations to cope with the contrast problem. Amongst others, an approach based on multi-resolution deformable models has been considered [12], while Baudin et al. [13] presented a method that uses random walks for thigh muscle segmentation. Incorporation of multi-channel data can provide additional constraints, but has not been considered yet for muscle segmentation.

\subsection{Dynamical modeling}

In biomedical applications, simulations of human musculoskeletal dynamics are most commonly performed using multi-body models in which the musculoskeletal system is represented as rigid segments which articulate in connecting joints and are actuated on by muscle forces [14]. To keep the computational cost manageable, joints are modeled with idealized geometries, while the musculotendon units are defined as series of line segments that represent their effective lines of action by approximating the centroidal path between the muscle attachments [14]. Construction of the musculoskeletal model typically depends on scaling of a generic model that is based on average anthropomorphic measurements in cadavers according to body segment dimensions of the individual. Alternatively, MRI data can be used to construct the 
model from the 3D anatomy of bones and muscles, which allows to incorporate details such as bone deformities, resulting in more accurate subject-specific models $[2,4]$.

The simplifications made in multi-body models are not always adequate. When medical evaluation focuses on a single joint or muscle, more realistic modeling and simulation are required to increase the physical and mechanical details. Therefore, physically based approaches have been investigated to utilize continuum mechanics formulations for simulations of volumetric muscle deformation to account for the changing muscle geometry during contraction. Predicting the deformation allows to assess the internal displacements inside the muscle, which is difficult to measure experimentally. Several numerical approaches exist to solve the resulting partial differential equations, of which the finite element (FE) method has been widely adopted [15]. Subject-specific modeling approaches based on medical imaging have been proposed recently in, e.g. [16,17]. However, subject-specific FE simulation studies of muscle function so far have only considered simple prescribed joint motions as boundary conditions [16,18], while distributions of activation level has been typically assumed to be uniform [16] or approximated from surface electromyogram (EMG) measurements [18].

\subsection{Knee joint modeling}

The knee joint plays an important role in body movement, involving multiple interactions between bones, cartilages, menisci, ligaments, tendons and muscles. FE analysis has been extensively used in the past years to understand and predict biomechanical behavior of knee articulation using 3D computational models. Several constitutive models as proposed in, e.g. [19,20] have been developed to simulate and investigate the mechanical response of the soft tissues in the knee (e.g. menisci, cartilage or ligaments). These studies typically use a generic anatomical knee model to demonstrate the stress-strain relationships for idealized kinematics that represent the range of motion during motor tasks.

Only a limited number of studies such as [21,22] have been dedicated to FE knee models that combine both subjectspecific anatomy and kinematical measurements. This is in part due to the time- and work-intensive process that is needed to create a complete subject-specific model, comprising medical image segmentation, volumetric meshing and acquisition of kinematics data. Despite many studies reporting on the knee joint, the combined mechanical behavior of knee components during normal and pathological movements has not been fully uncovered, limiting the medical applications. Moreover, a 3D subject-specific model that considers both the connective soft tissues and muscles has not been yet presented in the literature. However, a complete understanding of the knee joint functionality needs to address and correlate different biological scales, from cellular to organ level, especially when considering pathological progression. Recently, a multiscale modeling approach was proposed to investigate the coupling between macroscopic joint articulation and the microscopic deformations of chondrocyte cells in the cartilage [23]. The proposed method considered continuum FE models for both the articulation model and a tissue model with embedded chondrocyte cells, but did not account for stochastic cellular processes.

\section{Multimodal physiological model of knee articulation}

Simulation of physiological joint articulation encompasses a combination of anatomical, kinematical and physical modeling, which allows to identify underlying relations. This is exemplified by previous research efforts at MIRALab, which have culminated in an innovative modeling workflow to reconstruct subject-specific 3D articulations of the hip joint and analyze and visualize the functional implications during large movements [3]. In summary, the workflow starts from the segmentation of bones and soft tissues in MRI acquisitions, for which an efficient approach was developed based on dynamic deformable models. The segmented bone structures (femur and pelvis) are subsequently registered with body scan and optical motion capture data. This allows to compute the joint motion from the marker movements, for which a robust soft tissue artifacts (STA) correction algorithm was devised that accounts for geometrical constraints to avoid non-physiological dislocation and bone penetration. The reconstructed joint kinematics form the input to calculate the deformation and mechanical stress in the surrounding cartilage structures in FE simulations using a fast and accurate first-order corotational scheme. As a result, this modeling workflow allowed to detect the hip joint regions most affected by collisions and high cartilage stress in professional dancers, which correlated with radiological analyzes.

This modeling workflow demonstrates how integration between data modalities combined with virtual simulations can enhance medical profiling of patients. However, physiological content of the virtual articulation model can be further enhanced by increasing the spectrum of data modalities, but this poses challenges related to data fusion and coupling between simulations. Here, we present a multimodal approach focusing on the knee articulation that infuses additional data modalities not considered previously. As shown in Fig. 1, the broad spectrum of modalities provides a complementary mix of subject-specific data acquisitions performed in clinical practice and detailed experimental measurements. In the next sections, the synergy between the different modalities in the construction of virtual models of knee articulation on different scales is described. 

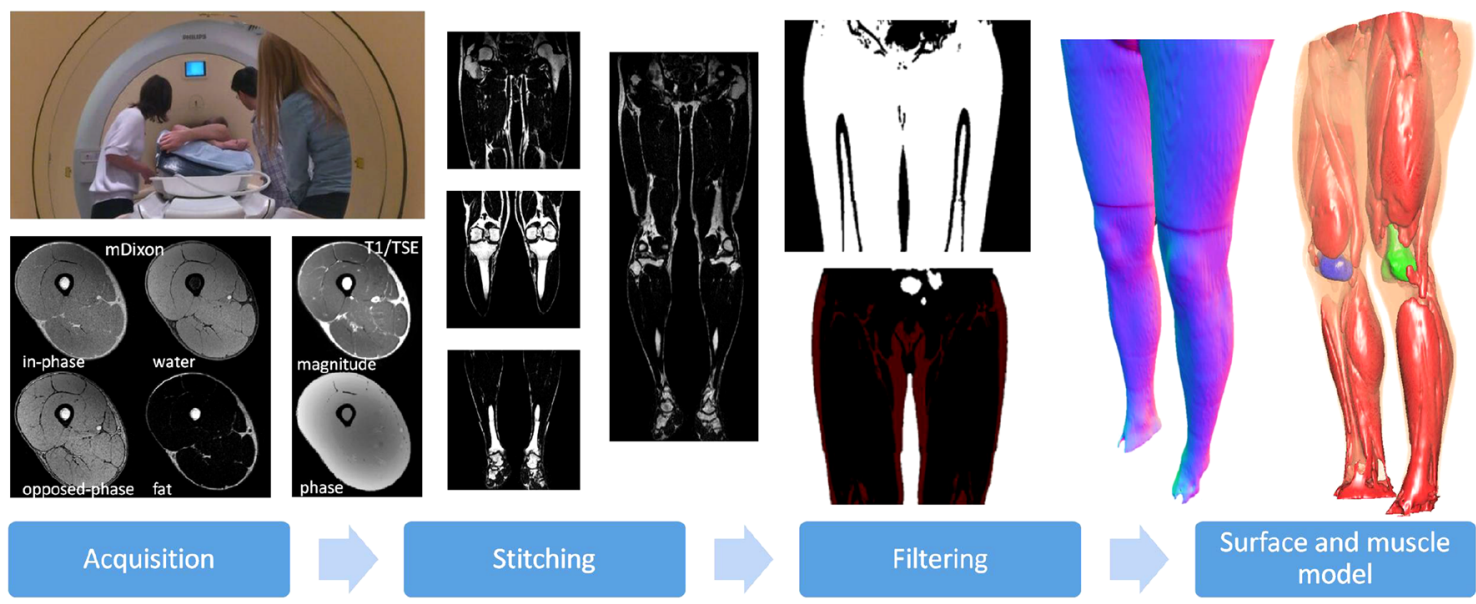

Fig. 2 The complete pipeline for acquisition and post-processing of multi-channel MRI images. Acquisition is followed by consecutive filtering steps to separate air, fat and muscles

\subsection{Anatomical modeling}

The differences in appearances and contrast obtained with different MRI protocols can potentially be used to enhance deformable model segmentation. Therefore, we consider two type of imaging sequences, T1 Turbo Spin Echo (TSE) and mDixon to acquire images of the lower limbs. As shown in Fig. 2, six different channels are obtained. The scans give different magnitude-based images from mDixon and T1/TSE sequence. The mDixon magnitude images are the in-phase image $I_{\mathrm{I}}$, the opposed-phase image $I_{\mathrm{O}}$, the fat image $I_{\mathrm{F}}$ and the water image $I_{\mathrm{W}}$. The T1/TSE scan produces an magnitude image $I_{\mathrm{M}}$ and a phase image $I_{\mathrm{P}}$.

The resulting multi-channel MRI data sets pose considerable challenges in terms of efficient managing of data flows during segmentation calculations which are not specifically considered in current systems for medical image segmentation research. Therefore, a dedicated computational framework for multi-channel deformable model segmentation was implemented that builds upon current state-of-the-art open source software packages. The framework has supporting mechanism for model loading and handling using the OpenMesh library ${ }^{1}$. The image processing component is based on ITK $^{2}$, which provides many advanced filtering algorithms and has special features to load multiple image sets and to perform calculations. An interface layer was created using the Lua language to make the complete framework scriptable, which allows for fast changes and the integration into more complex setups.

The segmentation process of the muscles consist of deforming a generic mesh model into the shape of the muscle using appropriate image forces. A combination of dif-

\footnotetext{
${ }^{1}$ http://www.openmesh.org.

2 http://www.itk.org.
}

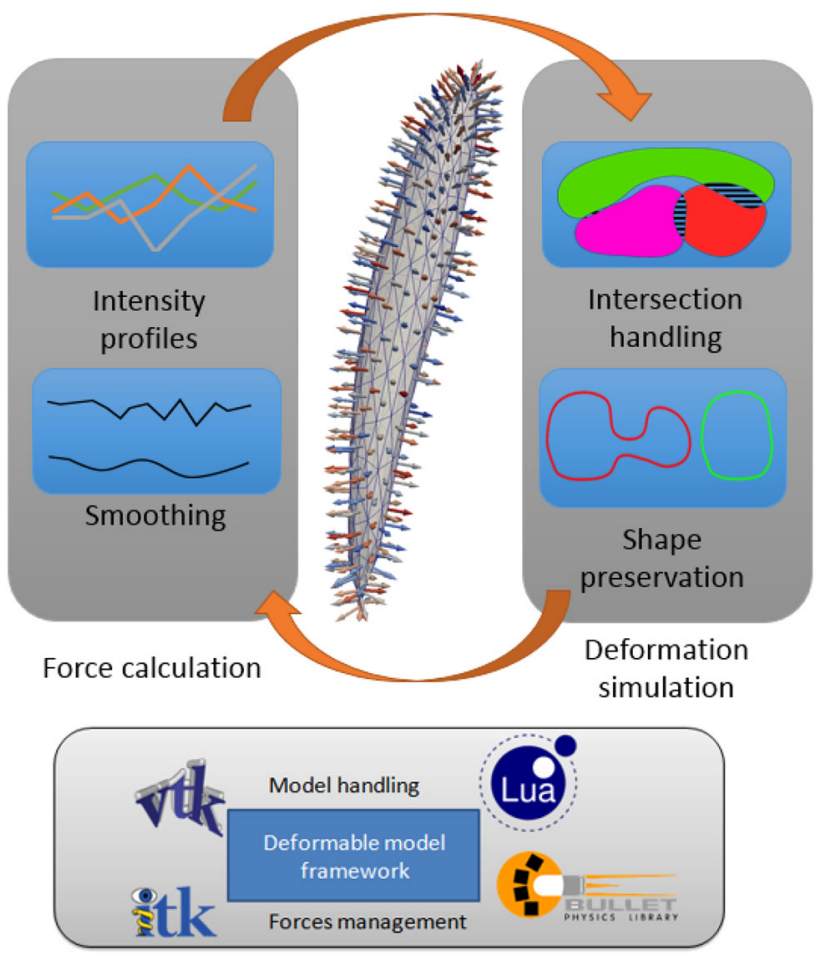

Fig. 3 Overview of the main components of the iteration process of the deformable model comprising forces calculation and deformation simulation. A dedicated framework allows for an efficient integration between the different components

ferent forces is used: growing forces along mesh normals to prevent model stagnation at low image information areas and smoothing forces that impose continuity and curvature constraints. The actual image information can be exploited using gradients, intensity profiles and texture information. In our approach we combine the acquired images $\left(I_{\mathrm{I}}, I_{\mathrm{O}}\right.$, $\left.I_{\mathrm{F}}, I_{\mathrm{W}}, I_{\mathrm{M}}, I_{\mathrm{P}}\right)$ as input to simultaneously compose multichannel image force based on the differences in image visi- 
bility. The deformable model segmentation process has two main components: The construction of forces that guide the deformation and the integration in a simulation to balance these forces. The iterative process is shown in Fig. 3. The force balancing in the simulation part deforms the mesh models as soft bodies and manages properties like stiffness, damping and general shape preservation. Collision detection plays an important role as many of the muscles are touching each other. In our deformable model framework, algorithms from the Bullet Physics ${ }^{3}$ engine for physical simulations are implemented to manage the multiple collision detections efficiently.

\subsection{Soft tissue fiber modeling}

A multimodal strategy for the physical simulation of joint articulation involves a coupling of subject-specific anatomical models with biomechanical models of the tissue material behavior. The material laws are typically described in local coordinate systems with reference to microstructural directions of material anisotropy. In the musculoskeletal soft tissues such as muscles, tendons, ligaments and menisci, the anisotropy is determined by the orientations of the fiber bundles. The trajectories of these bundles define the force directions in muscles while reinforcing the tensile strength in the connective tissue structures. Therefore, an adequate representation of the fiber arrangement within the anatomical model is required to enable realistic simulations of deformation.

Because in vivo 3D measurements are difficult the achieve, computational approaches have been devised to construct mathematical representations of fiber bundle arrangements in 3D anatomical models for physical simulations. Different methods were proposed with application for muscles $[16,17]$, ligaments [24] and menisci [25]. However, most of these approaches involve geometrical simplifications or assumptions pertaining to the anatomical shape of a specific tissue organ, such that the proposed methods are difficult to generalize for the variability in soft tissue anatomy. Consequently, a multimodal construction of the knee joint articulation which involves multiple soft tissues would require the implementation of plural methods based on disparate numerical concepts which is impractical to manage. The main musculoskeletal soft tissues share a common hierarchical bundled organization of fibers running between attachments, which has physical properties that can be represented by a Laplacian vector field as demonstrated for skeletal muscles [26]. Therefore, we propose to extend the application of the Laplacian based approach as a collective methodological basis to model the 3D fiber arrangements in anatomical models of the various musculoskeletal soft tissues.

\footnotetext{
${ }^{3}$ http://bulletphysics.org/.
}

The Laplacian method involves solving the differential Laplace equation in the anatomical model which requires the generation of a volumetric mesh as for the construction of a FE model. The attachment areas are indicated on the surface and a uniform flux can be applied as Neumann boundary conditions, which allows for the generation of physically plausible fiber directions in the absence of experimental data as demonstrated in skeletal muscle models in [26]. Solutions of the fiber orientation vectors can be obtained using common finite element or finite volume methods, but the latter also calculates the corresponding element flux values in the mesh. This allows for a robust tracing of the fiber bundle trajectories between the attachments, contrary to tracing methods based on vector interpolation.

Some tissues are characterized by multiple fiber families. In the knee menisci, for example, the fibers are organized in circumferential bundles, with sparse bundles in the radial directions. Therefore, meniscus tissue is often modeled as an orthotropic material, which requires the definition of three perpendicular directions that reflects the multiple fiber anisotropy. The definition of the circumferential and radial fiber orientations can be obtained by applying the Laplacian method with the attachments defined at, respectively, the horns of the menisci and at the internal and external surfaces as illustrated in Fig. 4 (indicated in red and green, respectively).

The feasibility of the Laplacian approach to generate fiber trajectories in anatomical models of the wide range of musculoskeletal soft tissue structures is illustrated in Fig. 4. Therefore, it can provide a collective methodological basis that allows for a comprehensive implementation, which benefits from fast solving strategies for the linear Laplace equation. This is particularly useful for surgical simulators which are valuable additions to traditional teaching methods, but currently available devices require further enhancement of the realism in the soft tissue behavior, which can be achieved by incorporating representations of fiber orientations to model the anisotropy. In future steps, the Laplacian approach can be further developed through detailed comparison with subjectspecific measurements, when a reference method or data set has been established, allowing for optimizing the boundary conditions at the attachment areas to match or smooth experimental data.

\subsection{Dynamic joint articulation modeling}

Physical simulation of joint articulation requires the definition of external forces as boundary conditions. A common approach is to estimate muscle and joint reaction forces in dynamic simulations based on multi-body models [14], limiting the muscle action to discrete point locations. However, this only gives adequate approximations for muscles with narrow tendon insertions. Anatomical 3D representations are 
Fig. 4 Fiber trajectories generated with the Laplacian based approach as demonstrated in examples of multiple musculoskeletal soft tissues

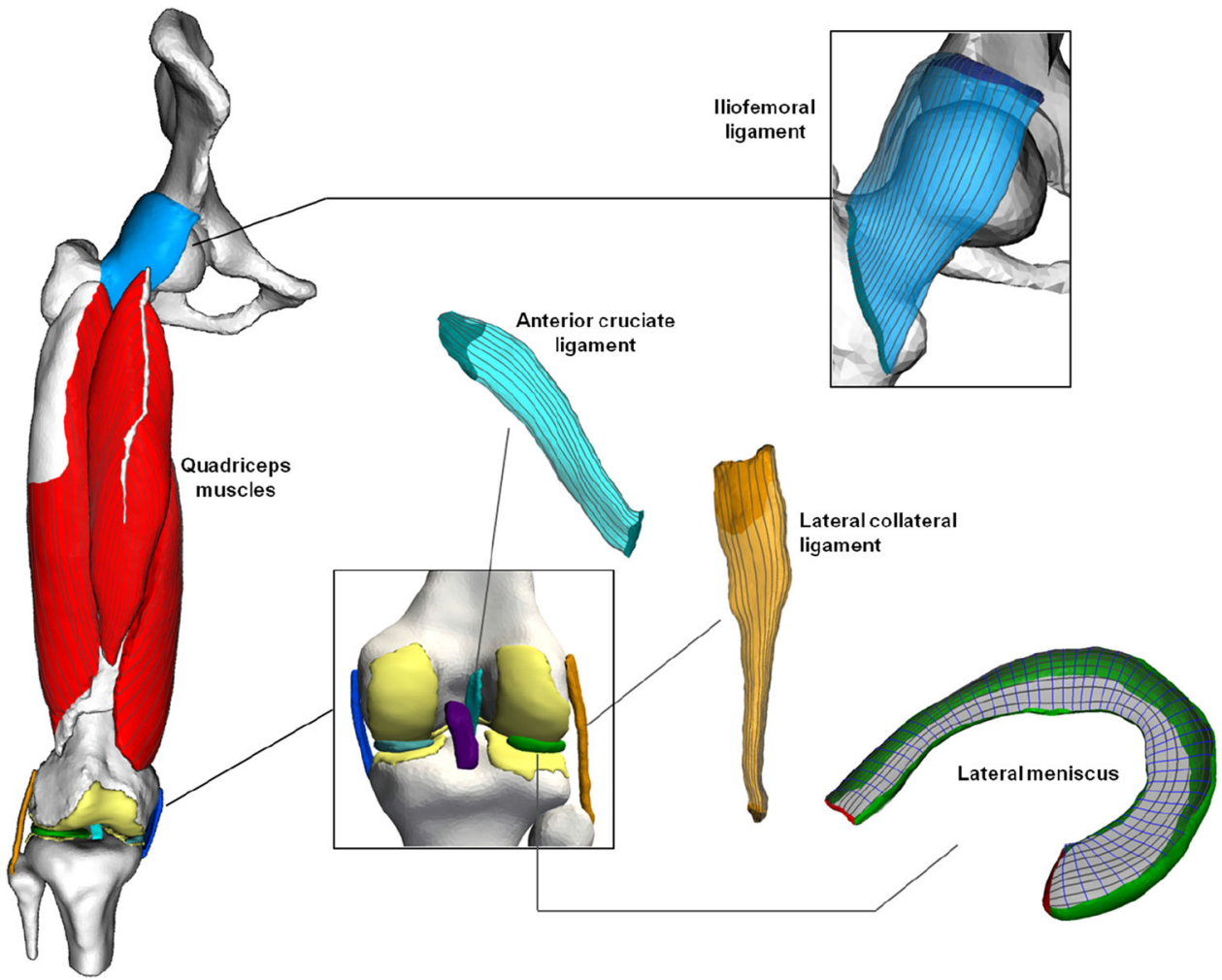

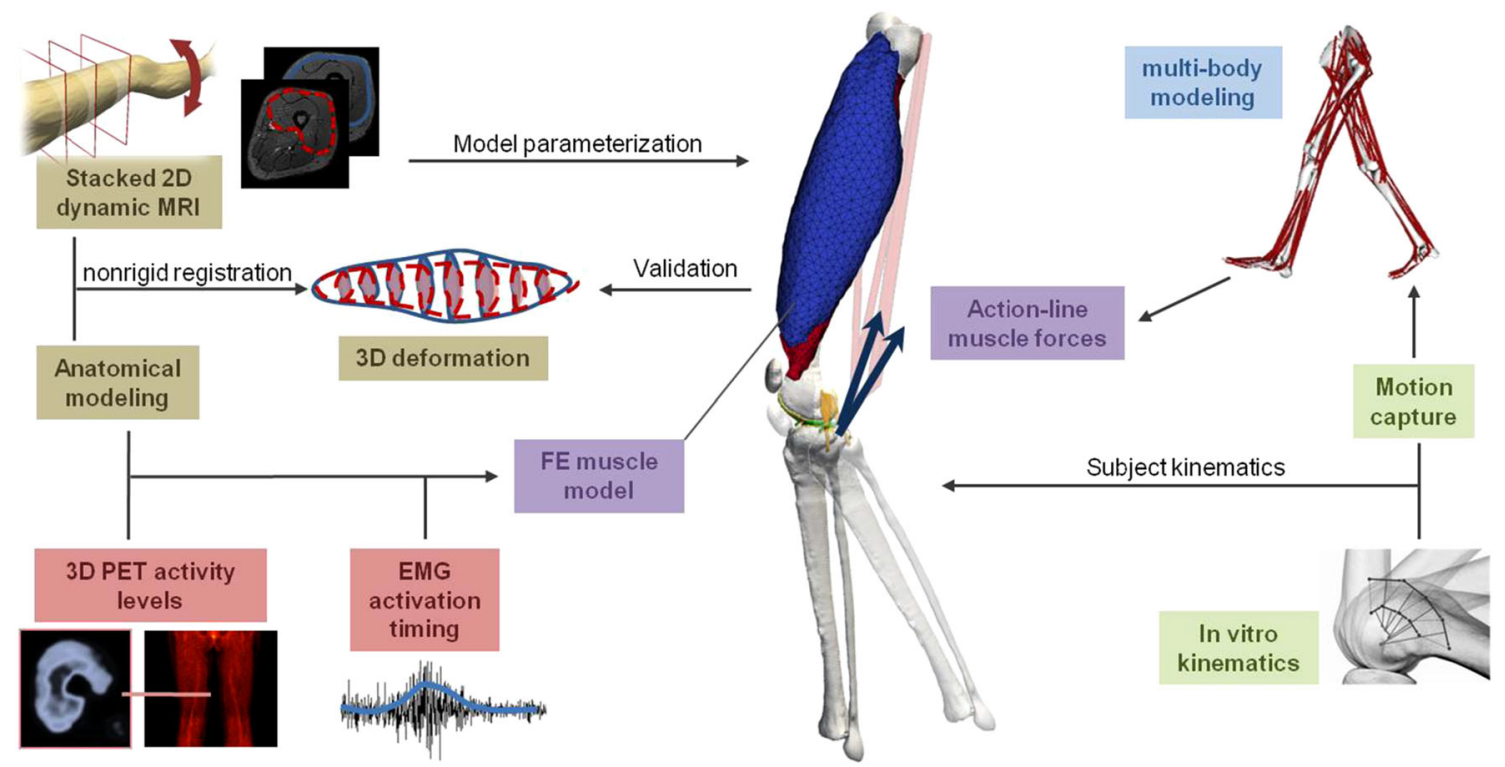

Fig. 5 Overview of the multimodal strategy for a dynamic knee articulation model. Volumetric FE representations of the large anterior quadriceps muscles with broad attachments are combined with multi-body line-action representations of the posterior hamstring muscles

more suited for muscles with broad attachments, but require computationally expensive methods such as FE to calculate the deformation and forces. Therefore, we propose an intermediate solution that accounts for muscles with narrow insertions using action-line representations while large muscles with broad attachments are incorporated through FE volumetric models, as illustrated in Fig. 5.
However, volumetric models require multidimensional input data to be used effectively. To obtain input of subjectspecific dynamical behavior, we consider a fusion with dynamic cine-MRI and positron emission tomography (PET) images to extract input parameters needed for simulations of volumetric muscle contraction (Fig. 1). Cine-MRI allows to visualize muscle deformation in the scanner, but only in 


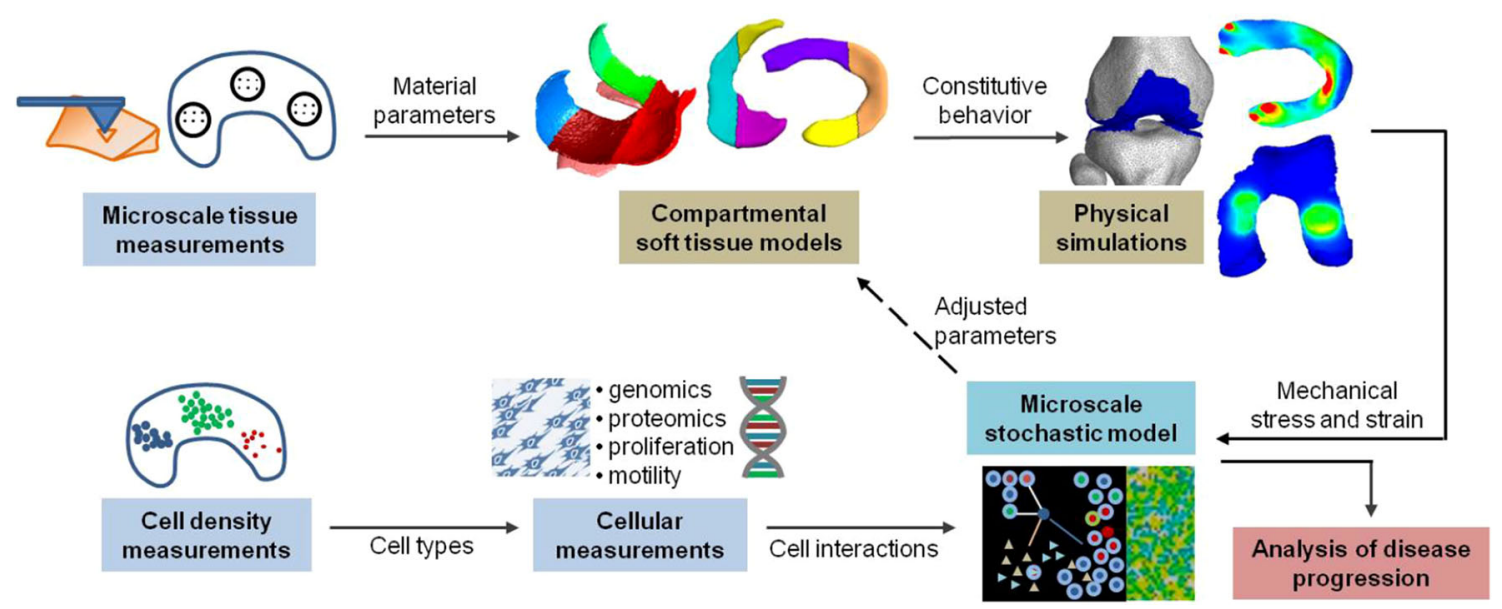

Fig. 6 Overview of the proposed workflow for constitutive modeling of knee cartilage and menisci tissue. Microscale tissue measurements allow for the construction of compartmental models with heterogeneous material parameters for physical simulations of mechanical stress and strain during articulation. Cellular measurements provide input for microscale models of cellular interactions that determine the evolution of diseased tissue behavior, resulting in changes of material parameters as feedback to the physical simulations
2D imaging planes to enable sufficient acquisition speed. Therefore, registration with segmentations from static MR images is required to extrapolate $3 \mathrm{D}$ deformation data, which can be used to optimize model parameters and for validation. PET imaging allows to measure volumetric distributions of metabolic activity in muscles after exercise [5]. Consequently, PET measurements offer the possibility to characterize the muscle activation levels in regional detail suitable for volumetric models. However, PET imaging does not give information about the temporal activation profile, which can be extracted from the EMG signals.

Input of subject-specific 3D knee kinematics remains difficult to acquire. The knee joint is a diarthrodal articulation that is only lightly constrained by the bone geometry, such that contact-based STA correction algorithms is likely less efficient than as demonstrated for the hip joint [3]. A potential solution is to combine cluster fitting of motion capture data with in vitro measurements of 3D knee kinematics, which provides a statistical constraint determined by the inter-subject variability. Additionally, these measurements provide a surrogate for subject-specific models in case the mobility is restricted in patients as illustrated in Sect. 4.

\subsection{Constitutive soft tissue modeling}

The majority of modeling studies rely on measurements obtained from experimental mechanical testing of in vitro cadaver or animal tissue samples to characterize the constitutive or material parameters [27]. These experiments typically quantify the macroscopic behavior of the soft tissue, assuming an overall uniform material behavior. However, the structural organization in biological soft tissues is commonly characterized by heterogeneous composition, which reflects the non-uniformity in mechanical loading conditions occurring during joint articulation. Accounting for this heterogeneity will provide a more detailed characterization of the local mechanical environment in different soft-tissue regions, which enables a more precise localization of high stress regions. This can be achieved by microscopic measurements (nano-indentation, dynamic mechanical analysis (DMA)) [6] to characterize the regional variations in material parameters. To incorporate these measurements, compartmental models of knee cartilage and menisci are developed with a subdivision according to clinical standard, such that locally measured material parameters can be assigned to each segment (Fig. 6). As such, physical simulations performed in these models allow to relate the local mechanical stress and strain to the distributions of cells and biomolecules in the different compartments. However, the mechanical properties will evolve during disease progression as determined by the physiological processes mediated by the cells in response to the mechanical loading. Indeed, the local tissue deformation influences a wide range of fundamental cell behaviors such as inflammation, proliferation or secretion of tissue matrix components such as collagen [6]. Therefore, introducing data of the cellular mechanobiology provides a means to construct predictive analyses that can aid in the prognosis of disease progression. To achieve this aim, stochastic rulebased methods as utilized in crowd simulations [28] provide a promising approach to model the microscale interactions between cells, molecules and therapeutic agents while offering a flexible solution to couple with the macroscale joint articulation simulations in a feedback loop as illustrated in Fig. 6. 


\section{Case study: knee stability analysis}

The ligaments have a major role in stabilizing the knee to ensure physiologic knee kinematics. The four major ligaments are anterior cruciate ligament (ACL), posterior cruciate ligament (PCL), medial collateral ligament (MCL) and lateral collateral ligament (LCL). A good understanding of the mechanical coordination between the knee ligaments is of great importance for preventing and treating ligament injuries. The objective of this study is to investigate whether a 3D finite element model reconstructed from MRI data (Fig. 7) combined with experimental in vitro data provides a suitable tool to evaluate this mechanical coordination as a virtual instrument for preoperative planning in ligaments surgery.

The model included the bony structures (femur, tibia, fibula) and all four major ligaments. The ligament segmentations were converted in tetrahedral volumetric meshes, while the bones were represented as rigid triangular polygon meshes. The ligaments were modeled as transversely isotropic hyperelastic Mooney-Rivlin behavior incorporating the fiber structure, which were generated with the Laplacian approach [26]. Simulations of knee stability were per-

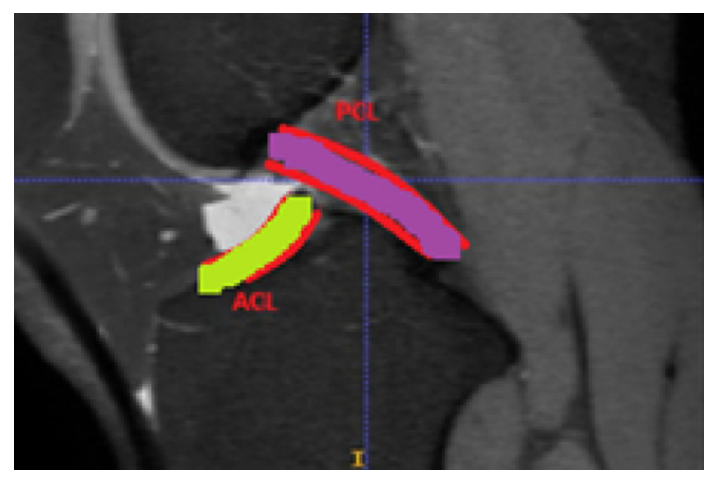

Fig. 7 High-resolution MRI image of the knee, with indication of the cruciate ligaments formed using the open source FEBio (v1.6) simulation environment [29].

Validation of the FE Model was performed by comparing in vitro measurements of anterior-posterior tibia translation kinematics with the translations predicted by the computational model.

The tibial reaction force in relation to the displacement in the anterior/posterior direction is shown in Fig. 8, which demonstrates a close agreement between the FE simulation results and the experimental measurements. Assessment of the mechanical stresses in the ligaments system under varying kinematical cases were investigated which included flexion from $0^{\circ}$ to $45^{\circ}$ (Flex) and internal rotation from $0^{\circ}$ to $15^{\circ}$ (IR).

These rotations were applied in the origin of the femur coordinate system and were in the physiologic range of walking [30]. The fiber strain was calculated in all cases to quantify the mechanical loading, of which the peak values under various angles are shown in Fig. 9c, d. In case of flexion, the highest value of the fiber strain was found for the MCL (around 0.31 ), suggesting that the MCL is the primary ligament to insure stability during flexion. During internal rotation, the fiber strain values were approximately three times lower compared to the values occurring during flexion. The highest fiber strain values (around 0.1 ) appeared in the collateral ligaments, responsible for the lateral knee stability. The highest values of fiber strain can be observed in the MCL, while the second ligament that supported internal/external rotations was found to be the LCL. Figure 9 also shows the main ligament stabilizers for each rotation: MCL is the main contributor for flexion while both MCL and LCL are important for internal rotation.

This study demonstrates that in vitro measurements provide a useful calibration for FE models in surgical planning and rehabilitation strategies. The anterior-posterior tibial translation as a measure of knee laxity is an important
Fig. 8 Tibial force versus anterior/posterior displacement in two in vitro cadaver experiments (S1 and S2) and simulated in the 3D FE model (FEM)
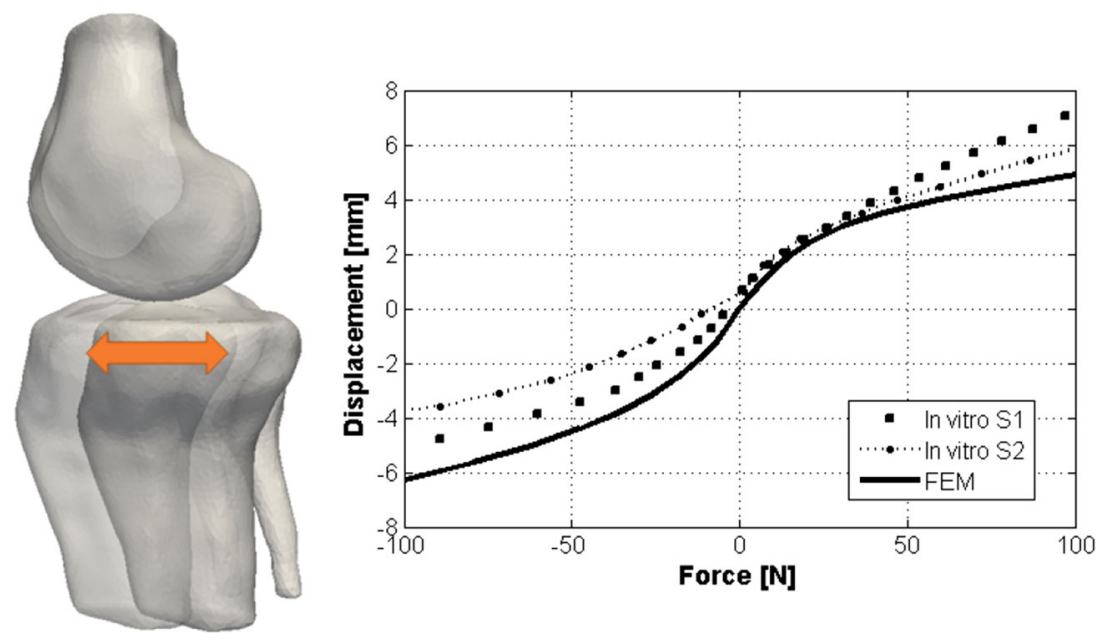


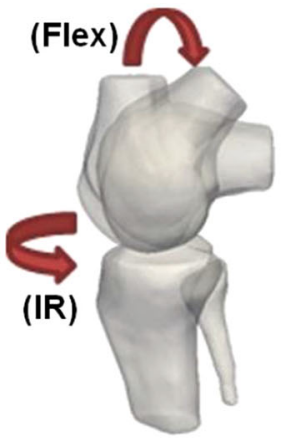

(a)

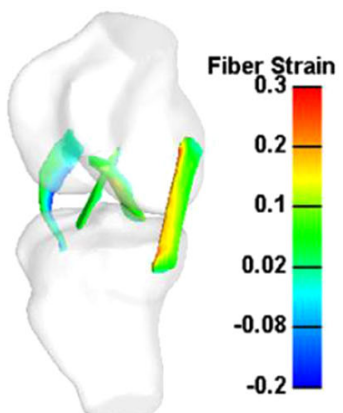

(b)

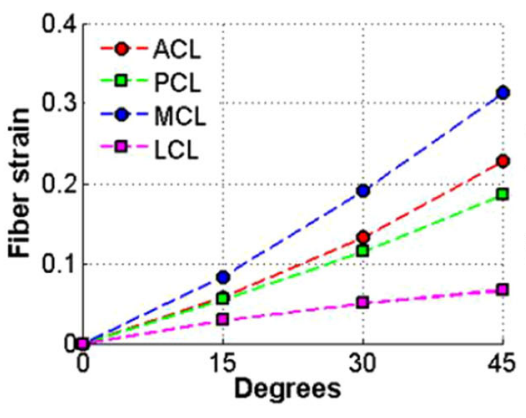

(c)

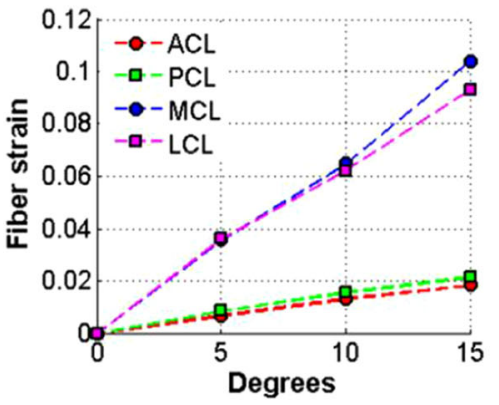

(d)

Fig. 9 FE simulations of fiber strain for flexion (Flex) and internal rotation (IR): a rotation orientations; b example of fiber strain distributions during flexion; peak fiber strains for $\mathbf{c}$ flexion and $\mathbf{d}$ internal rotation

medical indicator for the evaluation of knee instability [31]. The simulation results also demonstrate the mechanical loading interaction between all major knee ligaments under varying kinematical rotations, which has not yet been considered in detail previously. As illustrated in Fig. 9, the mechanical relations between the ligaments depends strongly on the range of knee rotations. To enable a comparison with results in previous studies, The motions have been varied separately. For surgical application, combinations of these motions can be systematically simulated to obtain a full kinematical map of the peak mechanical stresses for each ligament, allowing to detect the range of motion that imposes the most risk for further knee destabilization and injury. This information will allow the surgeon to refine the diagnosis and restore the primary function in the ligaments more effectively.

\section{Conclusion and future work}

The creation of virtual models of human articulation that can support medical diagnosis and treatment of musculoskeletal disorders remains a difficult challenge. Nowadays, physicians have a multitude of acquisition methods at their disposal for medical profiling of a patient's condition, but a deterministic approach for individual prognosis of treatment outcome or disease progression has not yet been reliably achieved. Evaluation through simulations in a virtual patient model provides a valuable instrument that can aid in achieving this goal, but requires investigation of approaches that can integrate data from multiple domains effectively to leverage the coupling between models of mechanical and physiological processes on different scales. In this work, we presented a multimodal strategy for virtual modeling of knee articulation that takes advantage of a complementary spectrum of data acquisitions, covering non-invasive subject-specific data from medical modalities, as well as experimental measurements to assess parameters that are difficult to determine in clinical practice. As demonstrated in a case study of knee stability evaluation, a multimodal approach offers extended opportunities for model validation and verification, which has to be considered carefully for medical applications. In future steps, the coupling between multiscale simulations will be further explored to investigate how the propagation of feedback between stochastic models of cellular processes and mechanical simulations of knee joint articulation can provide a scientific basis for prognosis of disease progression.

Acknowledgments This work has been funded by the EU FP7 MarieCurie ITN project MultiScaleHuman under Grant number 289897. We thank the University Hospital of Geneva in Switzerland, for providing the medical images, and the biomechanics laboratory LBB-MHH of the medical school in Hanover, Germany, for the experimental data of knee displacement. One of the authors, Nadia Magnenat Thalmann, is grateful to Humboldt Foundation to have allowed her to spend some time in Germany for collaboration with LBB-MHH and the Leibniz University in Hanover.

\section{References}

1. Rossi, R., Dettoni, F., Bruzzone, M., Cottino, U., D’Elicio, D.G., Bonasia, D.E.: Clinical examination of the knee: know your tools for diagnosis of knee injuries. BMC Sports Sci. Med. Rehabil. 3(1), 25 (2011)

2. Viceconti, M., Testi, D., Taddei, F., Martelli, S., Clapworthy, G., Jan, S.: Biomechanics modeling of the musculoskeletal apparatus: status and key issues. Proc. IEEE 94(4), 725-739 (2006)

3. Magnenat-Thalmann, N., Schmid, J., Assassi, L., Volino, P.: A comprehensive methodology to visualize articulations for the physiological human. In: 2010 International Conference on Cyberworlds (CW), pp. 1-8 (2010)

4. Scheys, L., Desloovere, K., Spaepen, A., Suetens, P., Jonkers, I.: Calculating gait kinematics using MR-based kinematic models. Gait Posture 33(2), 158-164 (2011)

5. Kalliokoski, K.K., Boushel, R., Langberg, H., Scheede-Bergdahl, C., Ryberg, A.K., Dossing, S., Kjaer, A., Kjaer, M.: Differential glucose uptake in quadriceps and other leg muscles during one-legged dynamic submaximal knee-extension exercise. Front Physiol. 2, 75 (2011). doi:10.3389/fphys.2011.00075 
6. McKee, C.T., Last, J.A., Russell, P., Murphy, C.J.: Indentation versus tensile measurements of young's modulus for soft biological tissues. Tissue Eng. Part B. Rev. 17(3), 155-164 (2011)

7. Bredno, J., Lehmann, T.M.T., Spitzer, K.: A general discrete contour model in two, three, and four dimensions for topologyadaptive multichannel segmentation. IEEE Trans. Pattern Anal. Mach. Intell. 25(5), 550-563 (2003)

8. Becker, M., Magnenat-Thalmann, N.: Deformable Models in Medical Image Segmentation. In: 3D Multiscale Physiological Human, 1st edn., chap. 4, pp. 81-106. Springer-Verlag, London (2014)

9. Chan, T.F., Sandberg, B., Vese, L.A.: Active contours without edges for vector-valued images. J. Vis. Commun. Image Represent. 11(2), 130-141 (2000)

10. Angelini, E.D., Imielinska, C., Jin, Y., Laine, A.F.: Improving statistics for hybrid segmentation of high-resolution multichannel images. In: Medical Imaging 2002: Image Processing, vol. 4684, pp. 401-411 (2002)

11. Geremia, E., Menze, B.H., Clatz, O., Konukoglu, E., Criminisi, A., Ayache, N.: Spatial decision forests for MS lesion segmentation in multi-channel MR images. In: Proceedings MICCAI 2010, pp. 111-118. Springer, Berlin Heidelberg (2010)

12. Gilles, B., Magnenat-Thalmann, N.: Musculoskeletal MRI segmentation using multi-resolution simplex meshes with medial representations. Med. Image Anal. 14(3), 291-302 (2010)

13. Baudin, P.Y., Azzabou, N., Carlier, P.G., Paragios, N.: Prior knowledge, random walks and human skeletal muscle segmentation. In: Proceedings MICCAI 2012, pp. 569-576. Springer, Berlin Heidelberg (2012)

14. Pandy, M.G., Andriacchi, T.P.: Muscle and joint function in human locomotion. Ann. Rev. Biomed. Eng. 12(1), 401-433 (2010)

15. Lee, D., Glueck, M., Khan, A., Fiume, E., Jackson, K.: Modeling and simulation of skeletal muscle for computer graphics: a survey. Found Trends Comput. Graph. Vis. 7(4), 229-276 (2012)

16. Blemker, S.S., Delp, S.L.: Three-dimensional representation of complex muscle architectures and geometries. Ann. Biomed. Eng. 33(5), 661-673 (2005)

17. Maurice, X., Sandholm, A., Pronost, N., Boulic, R., Thalmann, D.: A subject-specific software solution for the modeling and the visualization of muscles deformations. Vis. Comput. 25(9), 835842 (2009)

18. Fernandez, J.W., Hunter, P.J.: An anatomically based patientspecific finite element model of patella articulation: towards a diagnostic tool. Biomech. Model Mechanobiol. 4(1), 20-38 (2005)

19. Pena, E., Calvo, B., Martínez, M., Doblaré, M.: A threedimensional finite element analysis of the combined behavior of ligaments and menisci in the healthy human knee joint. J. Biomech. 39(9), 1686-1701 (2006)

20. John, D., Pinisetty, D., Gupta, N.: Image based model development and analysis of the human knee joint. Biomedical Imaging and Computational Modeling in Biomechanics. Lecture Notes in Computational Vision and Biomechanics, vol. 4, pp. 55-79. Springer, Netherlands (2013)

21. Yang, N.H., Nayeb-Hashemi, H., Canavan, P.K., Vaziri, A.: Effect of frontal plane tibiofemoral angle on the stress and strain at the knee cartilage during the stance phase of gait. J. Orthop. Res. 28(12), 1539-1547 (2010)

22. Shim, V., Mithraratne, K., Anderson, I., Hunter, P.: Simulating invivo knee kinetics and kinematics of tibio-femoral articulation with a subject-specific finite element model. In: World Congress on Medical Physics and Biomedical Engineering, IFMBE Proceedings 25(4), 2315-2318 (2010)

23. Sibole, S.C., Erdemir, A.: Chondrocyte deformations as a function of tibiofemoral joint loading predicted by a generalized highthroughput pipeline of multi-scale simulations. PLoS One 7(5), e37538 (2012)
24. Heimann, T., Chung, F., Lamecker, H., Delingette, H.: Subjectspecific ligament models: Toward real-time simulation of the knee joint. In: Computational Biomechanics for Medicine, pp. 107-119. Springer, New York (2010)

25. Erdemir, A.: Open knee: a pathway to community driven modeling and simulation in joint biomechanics. In: Proceedings ASME/FDA 2013 1st Annual Frontiers in Medical Devices. Washington, DC, USA (2013)

26. Choi, H.F., Blemker, S.S.: Skeletal muscle fascicle arrangements can be reconstructed using a laplacian vector field simulation. Plos One 8(10), e77576 (2013)

27. Kazemi, M., Dabiri, Y., Li, L.P.: Recent advances in computational mechanics of the human knee joint. Comput. Math. Methods. Med. 2013 (2013). doi:10.1155/2013/718423

28. Pelechano, N., Allbeck, J.M., Badler, N.I.: Controlling individual agents in high-density crowd simulation. In: Proceedings 2007 ACM SIGGRAPH/Eurographics Symposium on Computer, Animation, pp. 99-108 (2007)

29. Maas, S.A., Ellis, B.J., Ateshian, G.A., Weiss, J.A.: FEBio: finite elements for biomechanics. J. Biomech. Eng. 134, 011005-1, 011005-10 (2013)

30. Kadaba, M.P., Ramakrishnan, H.K., Wootten, M.E.: Measurement of lower extremity kinematics during level walking. J. Orthop. Res. 8(3), 383-392 (1990)

31. Kupper, J., Loitz-Ramage, B., Corr, D., Hart, D., Ronsky, J.: Measuring knee joint laxity: a review of applicable models and the need for new approaches to minimize variability. Clin. Biomech. 22(1), $1-13(2007)$

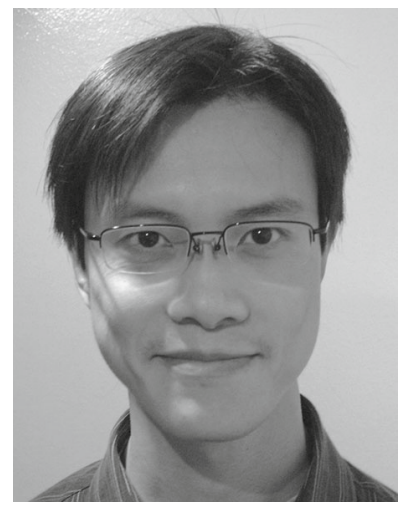

Hon Fai Choi is a postdoctoral researcher in MIRALab at the University of Geneva in Switzerland, currently working on the European FP7 MarieCurie MultiScaleHuman project. He received a M.Sc. in biology (2006) from Ghent University and a M.Sc. in physics (2002) and a $\mathrm{Ph} . \mathrm{D}$. in biomedical sciences (2011) from the Catholic University of Leuven in Belgium. He was a BAEF postdoctoral fellow at the University of Virginia, USA, in 2012. His research focus is on computational musculoskeletal modeling, with an emphasis on muscle biomechanics.

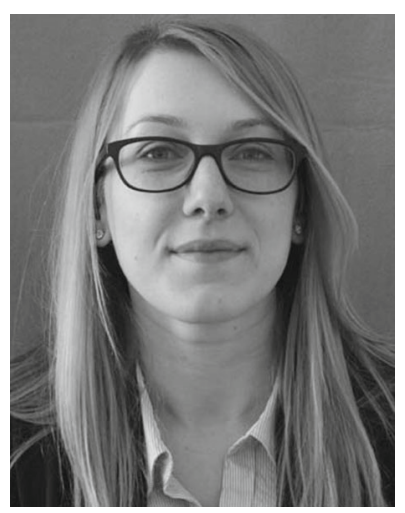

Andra Chincisan received a M.Sc. in Multimedia Technologies (2012) and a B.Sc. in IT and Telecommunications (2010) from the Technical University of Cluj-Napoca, Romania. She is a $\mathrm{Ph} . \mathrm{D}$. candidate and research assistant in MIRALab at the University of Geneva, Switzerland. She is currently working on the European FP7 Marie Curie MultiScaleHuman project. Her research is focused on musculoskeletal modeling of human articulations. 


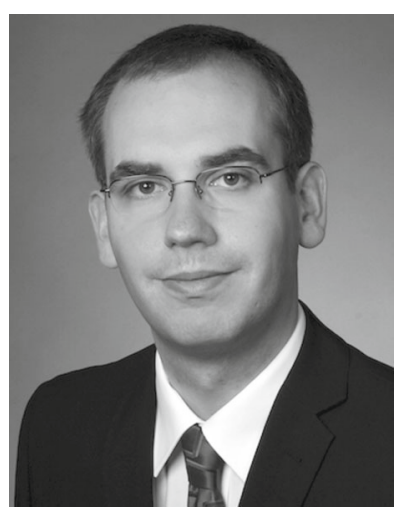

Matthias Becker is a Ph.D. candidate and research assistant in Computer Science at MIRALab at the University of Geneva. He obtained his M.Sc. in Computer Science from the Leibniz Universität Hannover, Germany, in 2011. His research interests include medical imaging techniques, image filtering, segmentation, and deformable models. He currently works on the EU FP7 Marie Curie research project MultiScaleHuman.

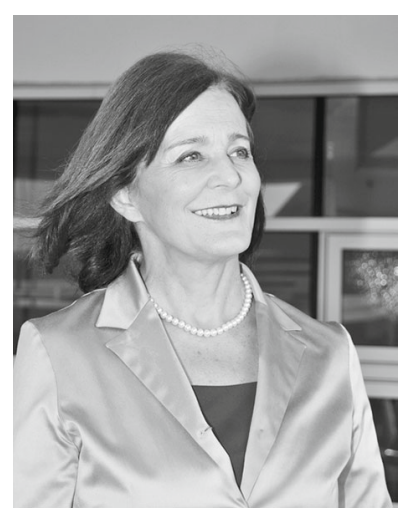

Nadia Magnenat-Thalmann has pioneered Virtual Humans research over the past 30 years with over 500 publications. She obtained degrees in various disciplines and a $\mathrm{PhD}$ in Quantum Physics from the University of Geneva. From 1977 to 1988 , she was a Professor at the University of Montreal. Since 1989, she has been Professor at the University of Geneva creating the interdisciplinary MIRALab. During her career, she has received numerous distinctions, recently, several Dr. Honoris Causa (Ottawa, Canada and Leibniz University of Hannover), the Eurographics Career Award and the Humboldt Research Award. She is Editor-in-Chief of The Visual Computer Journal published by Springer, and a life member of the Swiss Academy of Engineering Science. 